\title{
PREPARACIÓN DE CATALIZADORES BASADOS EN Cu-Mn PARA LA COMBUSTIÓN DE n-HEXANO
}

\author{
Gino Picasso $^{\mathrm{a}^{*}}$, Freddy Belleza ${ }^{\mathrm{a}}$, César Zavala ${ }^{\mathrm{a}}$, Alcides López ${ }^{\mathrm{a}}$, Rosario Sun Kou ${ }^{\mathrm{b}}$
}

\begin{abstract}
RESUMEN
Se preparó catalizadores basados en óxidos mixtos de $\mathrm{Cu}-\mathrm{Mn}$ ( relación molar $\mathrm{Cu} / \mathrm{Mn}$ de 0,33 a 3) por el método sol-gel de autocombustión para la eliminación de n-hexano. Se utilizó dos agentes de combustión: ácido cítrico y etilenglicol, para estudiar su influencia en el catalizador. Además, se preparó los óxidos simples de $\mathrm{Cu}$ y $\mathrm{Mn}$ con fines de comparación. La caracterización de los catalizadores se realizó mediante difracción de rayos X (XRD) y sorción de $\mathrm{N}_{2}$ (método BET). Todas las muestras presentaron una superficie específica preferentemente mesoporosa con áreas entre 4 y $50 \mathrm{~m}^{2} / \mathrm{g}$. Los difractogramas de los catalizadores mixtos mostraron una buena cristalinidad independiente de su composición, con la presencia de la fase espinela-hopcalita para los óxidos mixtos, a diferencia de los óxidos de $\mathrm{Mn}$ y $\mathrm{Cu}$ puros que presentaron picos atribuibles a $\mathrm{Mn}_{2} \mathrm{O}_{3}, \mathrm{Mn}_{3} \mathrm{O}_{4}$ y del $\mathrm{CuO}$, respectivamente. Las curvas de actividad medida como moles convertidos de COV por hora y por gramo de catalizador, considerando la superficie específica, mostraron que los catalizadores mixtos con mayor contenido en Mn fueron los más activos, adicionalmente la muestra preparada utilizando ácido cítrico presentó mayor actividad comparada con la obtenida con los óxidos simples, debido probablemente a una mejor superficie específica y estructura cristalina espinela-hopcalita obtenida.
\end{abstract}

Palabras clave: óxidos mixtos, $\mathrm{Cu}-\mathrm{Mn}$, eliminación de n-hexano.

\section{PREPARATION OF CATALYSTS BASED ON Cu-Mn FOR COMBUSTION OF n-HEXANE}

\begin{abstract}
Catalysts based on $\mathrm{Cu}-\mathrm{Mn}$ mixed oxides (with molar ratio $\mathrm{Cu} / \mathrm{Mn}$ in the range of 0,33 to 3 ) have been prepared by sol-gel method of self-combustion for removal of n-hexane. Two combustion agents, citric acid and ethylenglycol, were applied to study their influence in the final catalyst. Additionally, simple oxides have been synthesized using the same procedure for comparison reasons. The catalysts were characterized by X-ray diffraction (XRD) and sorption of $\mathrm{N}_{2}$ (BET method). All samples depicted surfaces, preferentially assigned to mesoporosity whose values ranged from 4 to $50 \mathrm{~m}^{2} / \mathrm{g}$. All XRD difractograms of mixed samples showed the presence of a good crystalinity indepently of composition, with the formation of spinnel-hopcalite phase meanwhile $\mathrm{Mn}$ and $\mathrm{Cu}$ simple oxide showed peaks attributed to $\mathrm{Mn}_{2} \mathrm{O}_{3}, \mathrm{Mn}_{3} \mathrm{O}_{4}$ and $\mathrm{CuO}$, respectively. Curves of activity, measured as number of VOC molecules converted per hour and per gram of catalyst, considering the specific surface, showed that mixed oxides with more Mn content were the best, additionally, the sample

a Laboratorio de Investigación de Fisicoquímica. Facultad de Ciencias. Universidad Nacional de Ingeniería. Av. Túpac Amaru 210. Rímac. Lima-Perú*gpicasso@uni.edu.pe

b Sección Química. Departamento de Ciencias. Pontifica Universidad Católica del Perú. Av. Universitaria 1801. San Miguel. Lima 32
\end{abstract}


prepared from citric acid was more active than the corresponding values to simple oxides, probably due to the better specific surface and the better spinel-hopcalite structure obtained.

Key words: mixed oxides, $\mathrm{Cu}-\mathrm{Mn}$, removal of n-hexane.

\section{INTRODUCCIÓN}

El gran número y diversidad de fuentes que emiten compuestos orgánicos volátiles (COV's), el comportamiento de cada COV individual y las grandes diferencias en la composición química, variaciones espaciales y temporales que experimentan las emisiones, han hecho que se incrementen las investigaciones de nuevos métodos con los cuales se pueda mitigar su concentración en el ambiente ${ }^{1}$. En el presente trabajo el n-hexano fue elegido como molécula modelo por su amplia aplicación en nuestro país como solvente en la industria de pinturas, tintes y pegamentos.

Los primeros esfuerzos encaminados a reducir la concentración de los COV's, estuvieron enfocados en el uso de catalizadores a base de metales nobles como Pd, Pt, sin embargo, el elevado costo de estos métodos llevó a buscar alternativas cuya aplicación fuera más económica. De esta forma, se empezaron a utilizar óxidos metálicos como catalizadores en la reacción de combustión de los $\mathrm{COV}^{\prime} \mathrm{s}^{2}$. En este campo, han sido publicados interesantes trabajos en sistemas que utilizan como base el óxido de manganeso, debido a los distintos estados de oxidación que puede tener el manganeso, la oxidación de los COV's sobre su superficie se hace bastante factible ${ }^{3-6}$. La presencia de otro metal en el sistema mixto con manganeso ha potenciado sus propiedades oxidativas debido a un efecto de cooperación entre ambos metales ${ }^{7-11}$; entre los óxidos mixtos, que se vienen estudiando y que incluyen manganeso, se encuentra la hopcalita, $\mathrm{CuMn}_{2} \mathrm{O}_{4}$, la cual ha presentado buena actividad catalítica logrando conversiones importantes de $\mathrm{COV}^{\prime} \mathrm{s}$ a bajas temperaturas ${ }^{12-13}$. La actividad del catalizador depende fuertemente del método de preparación debido a que en esa etapa se le puede proporcionar al catalizador características muy importantes, como una alta área superficial, una fase cristalina estable y resistencia a altas temperaturas ${ }^{14-16}$.

La parte más importante en toda reacción catalítica es el catalizador, el cual debe ser activo, selectivo a la reacción de interés, estable, económico y de fácil accesibilidad. La aplicación de óxidos de $\mathrm{Cu}-\mathrm{Mn}$ presenta una singular característica de introducir diversos cationes metálicos superficiales con diferentes estados de oxidación, lo que permite desarrollar sistemas mixtos con gran variedad de sitios ácidos superficiales. La aplicación de óxidos mixtos de $\mathrm{Cu}-\mathrm{Mn}$ en la remoción de n-hexano, constituye una tecnología novedosa de gran interés en nuestro país, considerando la abundancia y fácil accesibilidad de ambos metales de partida. La preparación de catalizadores basados en $\mathrm{Cu}-\mathrm{Mn}$, que sean muy activos y estables para la eliminación de n-hexano, es el principal objetivo de este trabajo.

\section{Materiales de partida}

\section{PARTE EXPERIMENTAL}

Los materiales de partida fueron los siguientes:

1. Nitrato de cobre (II) trihidratado, $\mathrm{Cu}\left(\mathrm{NO}_{3}\right)_{2} \cdot 3 \mathrm{H}_{2} \mathrm{O} 99.5 \%$ (Merck, $\mathrm{PF}=241 ; 60 \mathrm{~g} / \mathrm{mol}$ ).

2. Nitrato de manganeso (II) tetrahidratado, $\mathrm{Mn}\left(\mathrm{NO}_{3}\right)_{2} \cdot 4 \mathrm{H}_{2} \mathrm{O} 98.5 \%$ (Merck, $\mathrm{PF}=$ $251.01 \mathrm{~g} / \mathrm{mol})$.

3. Etilenglicol, $\mathrm{HOCH}_{2} \mathrm{CH}_{2} \mathrm{OH} 99.5 \%$ (Merck, $\left.\mathrm{PF}=62,07 \mathrm{~g} / \mathrm{mol}\right)$.

4. Ácido nítrico, $\mathrm{HNO}_{3} 15 \mathrm{M}$ (Merck, $\left.\mathrm{PF}=63 \mathrm{~g} / \mathrm{mol}\right)$.

5. Ácido cítrico, $\mathrm{C}_{6} \mathrm{H}_{8} \mathrm{O}_{7} 99,5 \%$ (Scharlau, $\left.\mathrm{PF}=192,13 \mathrm{~g} / \mathrm{mol}\right)$.

6. Solución n-hexano, $\mathrm{C}_{6} \mathrm{H}_{14} 98 \%$ (Merck, $\mathrm{PF}=86,18 \mathrm{~g} / \mathrm{mol}$ ). 
7. Aire sintético, PRAXAIR, 4 ppm de humedad.

8. Agua destilada.

Se prepararon los óxidos mixtos de Cu-Mn por el método sol-gel de autocombustión, método por el cual un compuesto orgánico encapsula las partículas metálicas al punto de lograr un aumento brusco en la viscosidad del sistema formando un gel; los cationes metálicos son complejados y quedan atrapados en este gel, para luego ser sometidos a calcinación. Finalmente, el compuesto orgánico combustiona quedando como producto final el óxido correspondiente. Se utilizaron dos agentes de combustión: etilenglicol (Método EG) y ácido cítrico (Método CIT), considerando su amplia aplicación en la literatura ${ }^{17-21}$.

\section{Preparación de los catalizadores}

\section{Método EG.}

Se disolvieron cantidades específicas de las sales metálicas (tabla 1) en 10mL de agua, 40mL de $\mathrm{EG}$ y $10 \mathrm{~mL}$ de $\mathrm{HNO}_{3}$ (relación EG:nitrato= 4:1) ${ }^{18}$ siendo la concentración total de metales en la solución de $0,125 \mathrm{M}$. La concentración individual de cada metal fue determinado de acuerdo a la tabla 1 , dependiendo de la proporción $\mathrm{Cu} / \mathrm{Mn}$ que se quiere obtener. Inicialmente, se evaporó la mezcla obtenida a $60^{\circ} \mathrm{C}$ por 12 horas. Luego, se elevó la temperatura hasta $80^{\circ} \mathrm{C}$ y se dejó evaporar hasta la formación del gel. Se secó a $110^{\circ} \mathrm{C}$ y calcinó a $400{ }^{\circ} \mathrm{C}$ por 4 h. Las cantidades específicas utilizadas en la preparación de los catalizadores se muestran en la tabla 1.

Tabla 1. Peso de los componentes metálicos utilizado en la preparación por el método $\mathrm{EG}^{*}$

\begin{tabular}{cccccc}
\hline Catalizador & $\mathbf{C u} / \mathbf{M n}$ & $\mathbf{M}_{\mathbf{c u}}(\mathbf{g})$ & $\mathbf{N}_{\mathbf{c u}}$ (moles) & $\mathbf{M}_{\mathbf{M n}}(\mathbf{g})$ & $\mathbf{N}_{\mathbf{M n}}$ (moles) \\
\hline $\mathrm{CuO}_{\mathrm{x}}$ & $1: 0$ & 1,812 & 0,0075 & --- & --- \\
$\mathrm{MnO}_{\mathrm{x}}$ & $0: 1$ & --- & --- & 1,882 & 0,0075 \\
$\mathrm{Cu} 1 \mathrm{Mn} 1$ & $1: 1$ & 0,906 & 0,0038 & 0,941 & 0,0038 \\
$\mathrm{Cu} 1 \mathrm{Mn} 2$ & $1: 2$ & 0,604 & 0,0025 & 1,255 & 0,0050 \\
$\mathrm{Cu} 2 \mathrm{Mn} 1$ & $2: 1$ & 1,208 & 0,0050 & 0,628 & 0,0025 \\
$\mathrm{Cu} 1 \mathrm{Mn} 3$ & $1: 3$ & 0,453 & 0,0019 & 1,412 & 0,0056 \\
$\mathrm{Cu} 3 \mathrm{Mn} 1$ & $3: 1$ & 1,359 & 0,0056 & 0,470 & 0,0019 \\
\hline
\end{tabular}

*número total de moles $=0,0075$

\section{Método CIT.}

Se disolvió las sales metálicas en cantidades específicas (tabla 2) y el ácido cítrico en $150 \mathrm{~mL}$ de agua. Se mantuvo en reflujo por 2 horas a $80^{\circ} \mathrm{C}$. Se evaporó a la misma temperatura hasta formación del gel. Se secó a $105^{\circ} \mathrm{C}$ por 18 horas. Se calentó con una rampa de $5^{\circ} \mathrm{C} / \mathrm{min}$ hasta $500^{\circ} \mathrm{C}$, y luego por 2 horas más. Un resumen de la preparación de las muestras se presenta en la tabla 2. 
Tabla 2. Peso de los componentes metálicos utilizado en la preparación por el método CIT

\begin{tabular}{cccccccc}
\hline Catalizador & $\mathbf{C u} / \mathbf{M n}$ & $\begin{array}{c}\mathbf{M}_{\mathbf{c u}} \\
\mathbf{( g )}\end{array}$ & $\begin{array}{c}\mathbf{N}_{\mathbf{c u}} \\
(\mathbf{m o l e s})\end{array}$ & $\begin{array}{c}\mathbf{M}_{\mathbf{M n}} \\
\mathbf{( g )}\end{array}$ & $\begin{array}{c}\mathbf{N}_{\mathbf{M n}} \\
(\mathbf{m o l e s})\end{array}$ & $\begin{array}{c}* \mathbf{N}_{\text {ac. Cit. }} \\
(\mathbf{m o l e s})\end{array}$ & $\begin{array}{c}\mathbf{M}_{\text {ac. Cit. }} \\
\mathbf{( g )}\end{array}$ \\
\hline $\mathrm{CuO}_{\mathrm{x}}$ & $1: 0$ & 1,208 & 0,005 & --- & --- & 0,005 & 0,961 \\
$\mathrm{MnO}_{\mathrm{x}}$ & $0: 1$ & --- & --- & 1,255 & 0,005 & 0,005 & 0,961 \\
$\mathrm{Cu} 1 \mathrm{Mn} 1$ & $1: 1$ & 1,208 & 0,005 & 1,255 & 0,005 & 0,010 & 1,921 \\
$\mathrm{Cu} 1 \mathrm{Mn} 2$ & $1: 2$ & 1,208 & 0,005 & 2,510 & 0.010 & 0,015 & 2,882 \\
$\mathrm{Cu} 2 \mathrm{Mn} 1$ & $2: 1$ & 2,416 & 0,010 & 1,255 & 0,005 & 0,015 & 2,882 \\
$\mathrm{Cu} 1 \mathrm{Mn} 3$ & $1: 3$ & 0,604 & 0,003 & 1,883 & 0,008 & 0,010 & 1,921 \\
$\mathrm{Cu} 3 \mathrm{Mn} 1$ & $3: 1$ & 1,812 & 0,008 & 0,628 & 0,003 & 0,010 & 1,921 \\
\hline
\end{tabular}

*moles totales de metal $=$ moles de citrato $^{22}$

Las reacciones básicas del proceso fueron las siguientes:

Método CIT:<smiles>O=CC(O)C(O)C(C=O)C(=O)O</smiles>

Ácido Cítrico<smiles>[Y14]CC</smiles>

(Ecuación 1)

En la ecuación 1 se observa la formación de los enlaces de coordinación entre el ácido cítrico y los cationes metálicos ${ }^{23}$.

Método EG:

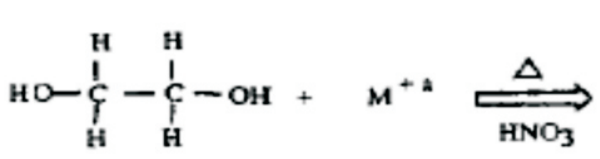<smiles>[M]O[C@@H](O)[C@@H](O)O[CH]</smiles><smiles>OCC(O)CO</smiles>

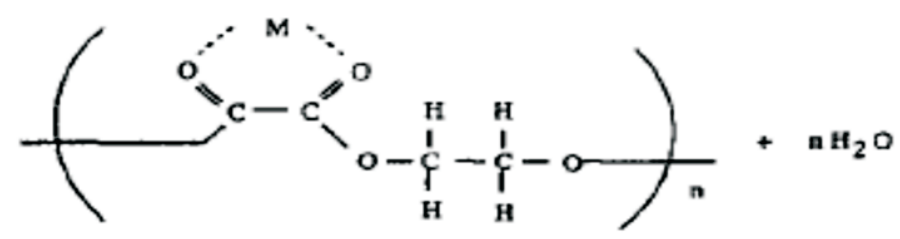

(Ecuación 2) 
En la ecuación 2 se muestra el mecanismo de reacción del proceso con etilenglicol ${ }^{18}$, como se $^{2}$ puede apreciar, el mecanismo de formación de las partículas de óxido metálico se diferencia de acuerdo al agente gelificante utilizado. En el caso del ácido cítrico, la formación del óxido se realiza a partir del complejo citrato mientras que en el caso del etilenglicol es a través de un polímero formado entre las moléculas de etilenglicol y el complejo metálico.

\section{RESULTADOS y DISCUSIÓN}

\section{Caracterización por sorción de $\mathrm{N}_{2}$ (método BET).}

Estos análisis fueron realizados en un equipo Micromeritics GEMINI-VII (serie t), utilizando $\mathrm{N}_{2}$ como gas de adsorción y He como gas de dilución y arrastre; previo a la medición, los catalizadores fueron desgasificados en una corriente de $\mathrm{He}$ a $250^{\circ} \mathrm{C}$ durante $2 \mathrm{~h}$.

Las isotermas de las muestras preparadas por el método CIT se presentan en la figura 1, como se observa todas las isotermas son de tipo IV correspondiente a sólidos micro-mesoporosos, con presencia preferente de mesoporosidad. Las isotermas de los óxidos mixtos y del óxido de $\mathrm{Cu}$ presentan ciclos de histéresis tipo $\mathrm{H} 1$ que es atribuido a la presencia de poros cilíndricos, con excepción de la muestra $\mathrm{MnOx}$ que presenta un ciclo de histéresis tipo H3, que revela la presencia de poros laminares. Las isotermas de las muestras preparadas por el método EG son similares como se aprecia en la figura 2, con la diferencia de que todos los ciclos de histéresis corresponden en este caso al tipo $\mathrm{H} 1$.

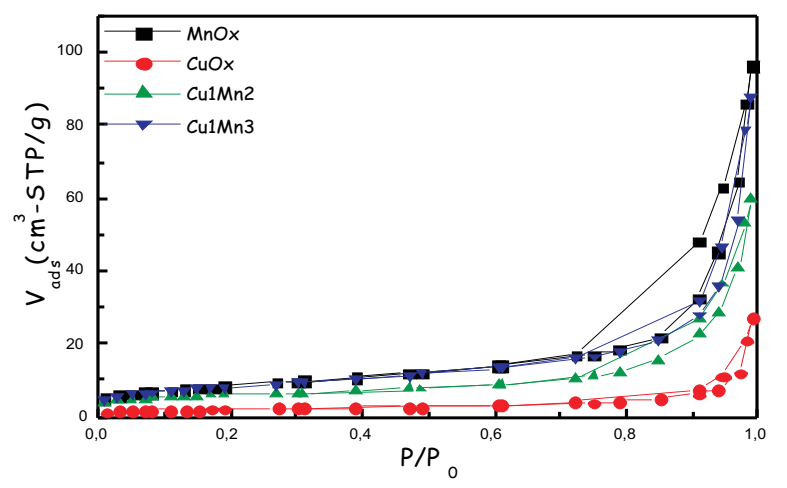

Figura 1. Isotermas de adsorción de los catalizadores preparados por el método CIT.

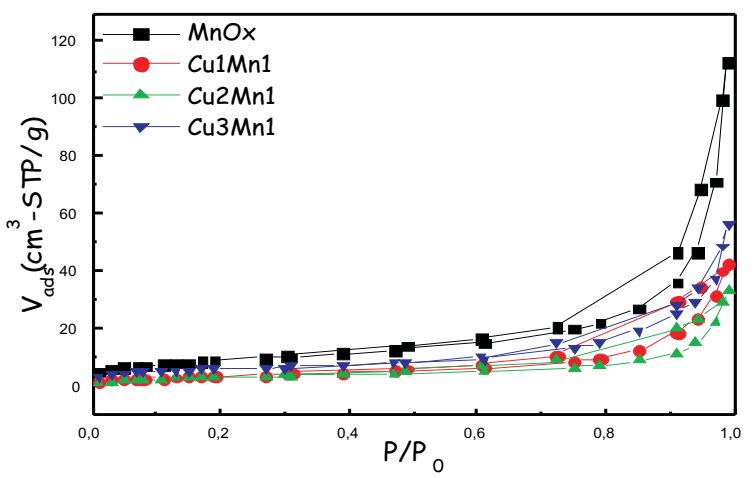

Figura 2. Isotermas de adsorción de los catalizadores preparados por el método EG. 
Los valores de superficie específica de los catalizadores preparados por el método $\mathrm{EG}\left(\mathrm{S}_{\mathrm{BET}^{-}}\right.$ EG) y método CIT ( $\left.\mathrm{S}_{\mathrm{BET}}-\mathrm{CIT}\right)$, así como del tamaño medio de partícula calculado por la expresión: $\mathrm{D}(\mathrm{nm})=6 / \mathrm{r} . \mathrm{S}_{\mathrm{BET}}$, donde $\mathrm{r}$ es la densidad, para las muestras preparadas por el método EG(D-EG) y CIT (D-CIT), se muestran en la tabla 3.

Tabla 3. Superficies específicas y tamaño medio de partícula de los catalizadores preparados por el método EG y CIT.

\begin{tabular}{ccccc}
\hline Catalizador & $\mathbf{S}_{\mathbf{B E T}}-\mathbf{E G}\left(\mathbf{m}^{\mathbf{2}} / \mathbf{g}\right)$ & $\begin{array}{c}\mathbf{D}-\mathbf{E G} \\
\mathbf{( n m})\end{array}$ & $\mathbf{S}_{\mathbf{B E T}}-\mathbf{C I T} \mathbf{( \mathbf { m } ^ { 2 } / \mathbf { g } )}$ & $\begin{array}{c}\mathbf{D - C I T} \\
\mathbf{( n m})\end{array}$ \\
\hline $\mathrm{MnO}_{\mathrm{x}}$ & 48,1 & 14,18 & 30,0 & 13,74 \\
$\mathrm{CuO}_{\mathrm{x}}$ & 4,7 & 13,88 & 7,3 & 24,42 \\
$\mathrm{Cu} 1 \mathrm{Mn} 1$ & 13,7 & 10,10 & 23,5 & 14,79 \\
$\mathrm{Cu} 1 \mathrm{Mn} 2$ & 33,3 & 13,47 & 20,1 & 14,69 \\
$\mathrm{Cu} 2 \mathrm{Mn} 1$ & 12,4 & 10,85 & 19,6 & 14,71 \\
$\mathrm{Cu} 1 \mathrm{Mn} 3$ & 22,3 & 14,42 & 29,1 & 16,63 \\
$\mathrm{Cu} 3 \mathrm{Mn} 1$ & 21,5 & 10,99 & 13,7 & 14,58 \\
\hline
\end{tabular}

Se puede observar en la tabla 3 que por ambos métodos, los óxidos de Mn presentan mayor área superficial en comparación con los óxidos mixtos; esto también se ha observado en otros trabajos $^{17-18}$. Se puede observar una variación del área superficial con la composición del óxido mixto. Para el caso del método EG (figura3), las superficies a partir del valor de $\mathrm{MnOx}$ muestran un decrecimiento con el incremento en la composición hasta composiciones de $\mathrm{Cu} / \mathrm{Mn}$ 1,0 (con excepción de la muestra $\mathrm{Cu} 1 \mathrm{Mn} 3$ ) donde se observa el valor máximo de área superficial lograda, luego el área superficial baja para la relación $\mathrm{Cu} / \mathrm{Mn}=2$, luego la tendencia aumenta con composiciones de $\mathrm{Cu} / \mathrm{Mn}$ de 3. En el caso del método CIT (figura 4), los valores del área también varían al aumentar la relación $\mathrm{Cu} / \mathrm{Mn}$, con una tendencia lineal. Las variaciones en los comportamientos probablemente se deban a diferencias en la reacción de combustión de estos compuestos, ya que esta etapa es determinante en la formación de la textura y porosidad del catalizador. Con ambos métodos se observa también una correlación aproximada entre la superficie específica y el tamaño medio de partícula, D(nm), como se observa en la figura 5 con los catalizadores preparados por el método CIT.

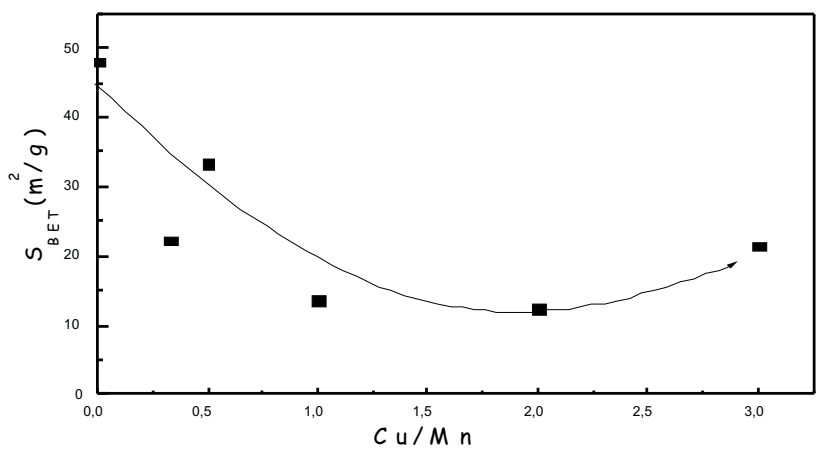

Figura 3. Correlación de los valores de la superficie $\mathrm{S}_{\mathrm{BET}}$-EG con la composición $\mathrm{Cu} / \mathrm{Mn}$. 


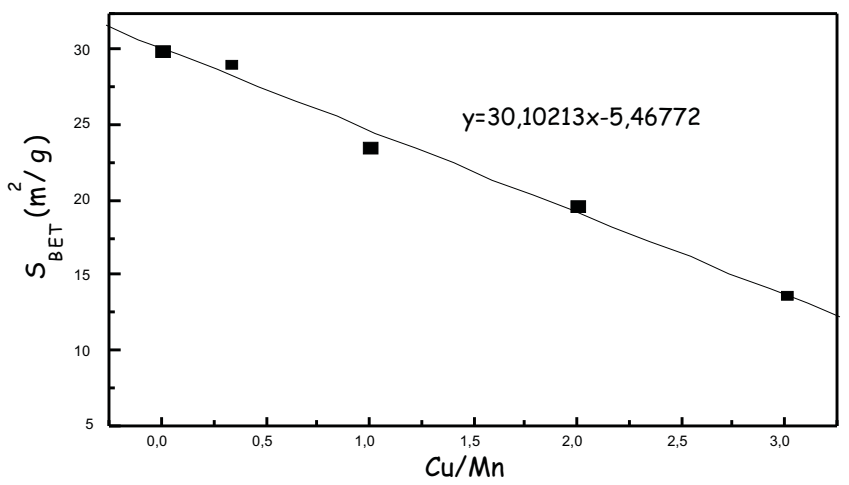

Figura 4. Correlación de los valores de la superficie $\mathrm{S}_{\mathrm{BET}}$-CIT con la composición $\mathrm{Cu} / \mathrm{Mn}$.

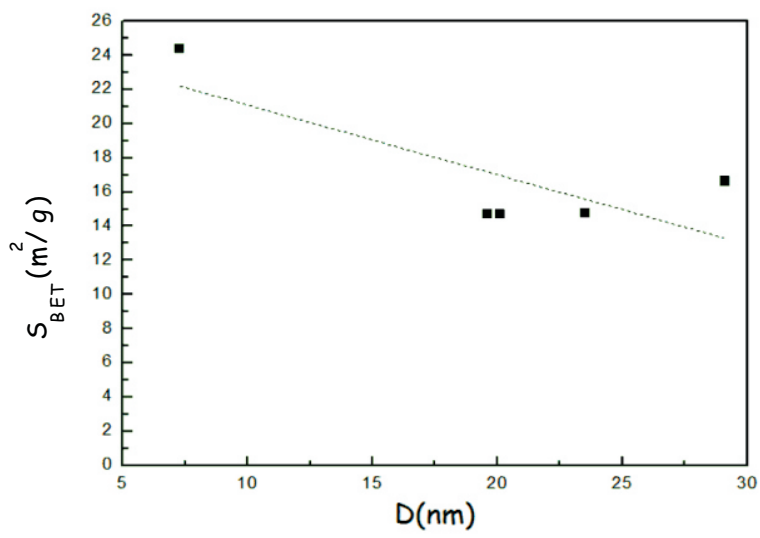

Figura 5. Correlación entre la superficie específica y el tamaño medio de partícula de los catalizadores preparados por el método CIT.

\section{Análisis por difracción de rayos X (XRD)}

El análisis XRD se realizó en un equipo RIGAKU modelo Miniflex con tubo de cobre y filtro de níquel; los ensayos se realizaron con $30 \mathrm{kV}$ y $15 \mathrm{~mA}$; se usó un monocromador de grafito con el objeto de seleccionar la radiación $\mathrm{CuK}$ del ánodo de cobre y se ha trabajado con ángulos de barrido entre 5 y $100^{\circ}$ a una velocidad de $0,04^{\circ} / \mathrm{s}$. 


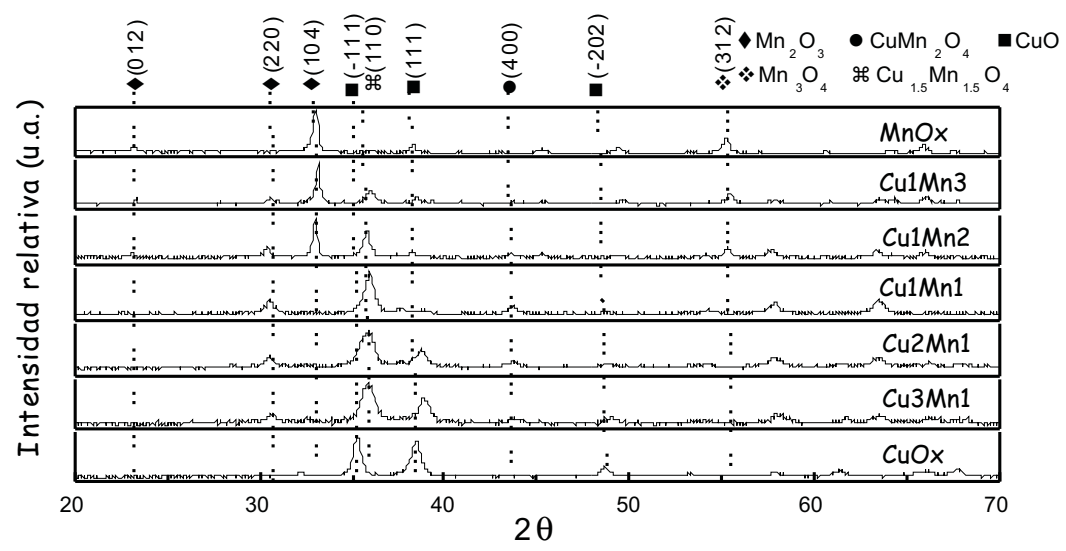

Figura 6. Difractogramas XRD de los catalizadores preparados por el método CIT.

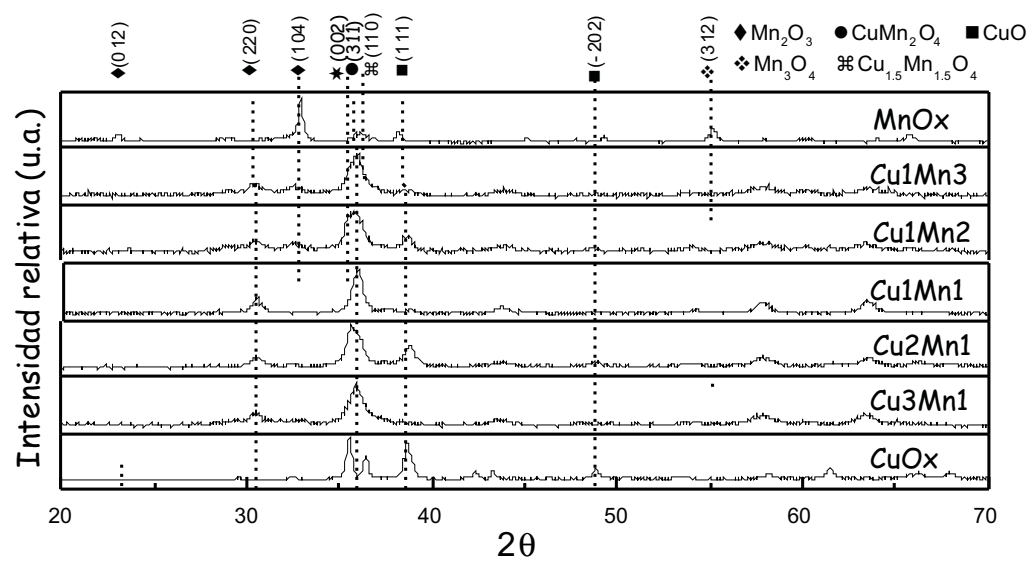

Figura 7. Difractogramas XRD de los catalizadores preparados por el método EG.

Según el análisis XRD, no se observó diferencias sustanciales en los difractogramas de las muestras preparadas con diferentes agentes de combustión: el acido cítrico (figura 6) y etilenglicol (figura 7). Los difractogramas de los catalizadores basados en óxidos simples muestran la presencia de los correspondientes óxidos simples, en el caso del $\mathrm{CuO}_{x}$, se observó el $\mathrm{CuO}$ y en el caso del $\mathrm{MnO}_{x}$, se apreció los picos correspondientes a $\mathrm{Mn}_{2} \mathrm{O}_{3}$ y $\mathrm{Mn}_{3} \mathrm{O}_{4}$. En los óxidos mixtos, la intensidad de los picos correspondientes a los óxidos simples se debilita a medida que disminuye la concentración del componente simple en el óxido mixto, por ejemplo el pico $\left(\mathrm{d}_{104}\right)$ del $\mathrm{Mn}_{2} \mathrm{O}_{3}$ con cierta intensidad en las muestras $\mathrm{Cu} 1 \mathrm{Mn} 3$ y $\mathrm{Cu} 1 \mathrm{Mn} 2$, desaparece a partir de la muestra $\mathrm{Cu} 1 \mathrm{Mn} 1$, al disminuir la composición del $\mathrm{Mn}$. En los 
difractogramas de las muestras mixtas se detectó además la presencia de picos correspondientes a las fases $\mathrm{CuMn}_{2} \mathrm{O}_{4}$ (hopcalita) y $\mathrm{Cu}_{1.5} \mathrm{Mn}_{1.5} \mathrm{O}_{4}$ en concordancia con otros trabajos $^{11-12}$.

\section{Evaluación de la actividad catalítica de los catalizadores.}

Las pruebas catalíticas se realizaron en un equipo de reacción que consiste de un reactor catalítico de lecho fijo de cuarzo de $60 \mathrm{~cm}$ de largo y $0,9 \mathrm{~cm}$ de diámetro interno con una frita fina de cuarzo dentro de un horno hexagonal, similar al descrito en un trabajo anterior ${ }^{14}$. En el interior del reactor, en contacto con el lecho fijo se colocó un termopar tipo K. Como flujo de alimentación al reactor se utilizó una corriente de aire saturada con n-hexano a $0^{\circ} \mathrm{C}$, esto se consiguió empleando una batería de 3 saturadores. Para el registro de salida de los productos de reacción se usó un cromatógrafo de gases (VARIAN modelo 450-GC) dotado de un detector FID, conectado en línea con el reactor. Se utilizó aire sintético $\left(79 \% \mathrm{~N}_{2}\right.$ y $\left.21 \% \mathrm{O}_{2}\right)$ como oxidante y gas de dilución. En todas las pruebas se trabajó con una concentración inicial de n-hexano de 2000 ppm y velocidad espacial respecto al peso del lecho catalítico (WHSV) de $80 \mathrm{~h}^{-1}$. La eficiencia de los catalizadores se han medido por las temperaturas de conversión de n-hexano al 50\% $\left(\mathrm{T}_{50}\right)$ y 95\% $\left(\mathrm{T}_{95}\right)$. Experimentos en blanco (en ausencia de componente activo) realizados previamente a la reacción de combustión, mostraron ausencia de actividad a temperaturas menores a $350^{\circ} \mathrm{C}$.

Las curvas de conversión versus la temperatura de reacción mostradas en la figura 8 , indican que todos los catalizadores preparados por el método CIT son capaces de lograr la combustión total de n-hexano. Se observa que el óxido de cobre presentó una conversión muy baja comparada con los otros óxidos utilizados, por el contrario, el óxido de manganeso presentó una actividad más alta y cercana a la de los óxidos mixtos; este comportamiento está asociado también con el área superficial del óxido de manganeso que fue mayor a las áreas superficiales de los demás óxidos mixtos, por esta razón la conversión de este óxido fue casi tan alta como la de los óxidos mixtos. Un comportamiento similar fue observado en las muestras preparadas por el método EG (figura 9), con la diferencia que todos los catalizadores tuvieron menor conversión que el correspondiente a $\mathrm{MnOx}$.

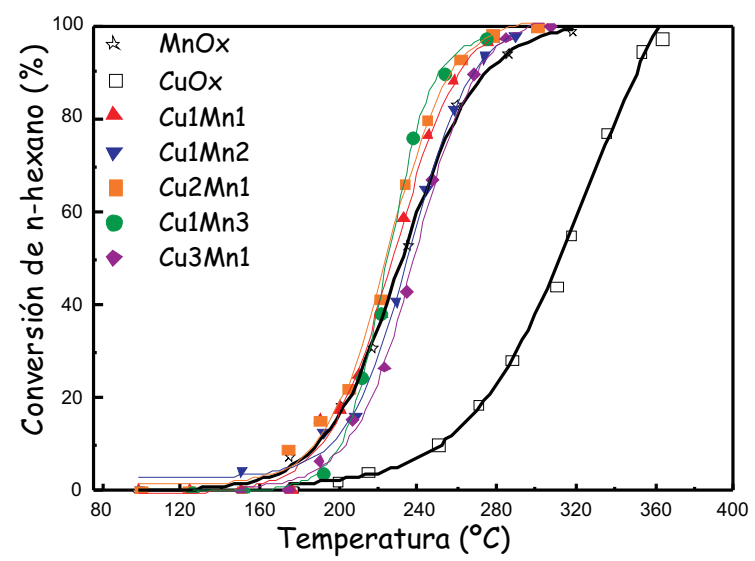

Figura 8. Curvas de conversión de los catalizadores preparados por el método CIT. 


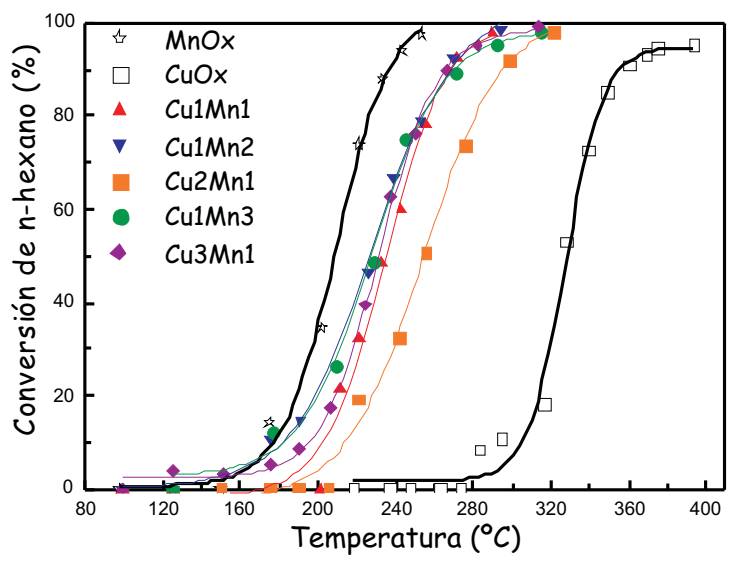

Figura 9. Curvas de conversión de los catalizadores preparados por el método EG.

La actividad, evaluada como velocidad específica expresa la cantidad de $n$-hexano convertido por unidad de tiempo. El cálculo de la velocidad específica $\left(\mathrm{v}_{\text {esp }}\right)$ a partir de la conversión expresada como fracción convertida de $\mathrm{n}$-hexano $(\mathrm{X})$ fue realizado mediante la ecuación 3:

$$
\mathrm{v}_{\text {esp }}=\left[\mathrm{X} \cdot\left(\frac{2000 \mu \mathrm{L}_{\mathrm{HEX}}}{\mathrm{L}_{\mathrm{AIRE}+\mathrm{HEX}}}\right) \cdot \mathrm{Q}\left(\frac{\mathrm{L}_{\mathrm{AIRE}+\mathrm{HEX}}}{\min }\right) \cdot \frac{1 \mathrm{~mol}(\mathrm{HEX})}{22400 \cdot 10^{3} \mu \mathrm{L}_{\mathrm{HEX}}} \cdot \frac{60 \mathrm{~min}}{\text { hora }}\right] \cdot \mathrm{S}_{\mathrm{BET}} \frac{\mathrm{m}^{2} \text { (cat) }}{\mathrm{g}(\text { cat })}
$$

(Ecuación 3)

Donde la concentración inicial de n-hexano es 2000 ppmV o $2000 \mu \mathrm{L}_{\text {hex }} / \mathrm{L}_{\text {are }}$, para un caudal Q de alimentación del aire saturado de $\mathrm{n}$-hexano de $0,1 \mathrm{~L}_{\text {airethexano }} / \mathrm{min}$ en condiciones estándar (es decir, $1 \mathrm{~mol}$ de hexano a $0^{\circ} \mathrm{C}$ ocupa un volumen de $22400 \times 10^{3} \mu \mathrm{L}$ ). Si la actividad se calcula en milimoles de n-hexano por hora de n-hexano para una superficie específica de $\mathrm{S}_{\mathrm{BET}} \mathrm{m}^{2}$ por gramo de catalizador, entonces:

$$
\mathrm{v}_{\text {esp }}=\left[\mathrm{X} \cdot \frac{2 \cdot 0,1 \cdot 60}{22400}\right] \cdot 10^{3} \cdot \mathrm{S}_{\mathrm{BET}} \frac{\mathrm{m}^{2}(\text { cat }}{\mathrm{g}(\text { cat })}
$$

(Ecuación 4)

La medición de la velocidad específica de los catalizadores permitió observar mejor las diferencias en actividad entre los catalizadores mixtos preparados por los métodos CIT (figura 10) y EG (figura 11). Como se observa en la figura 10, el catalizador $\mathrm{Cu} 1 \mathrm{Mn} 3$ convierte mayor cantidad de n-hexano por unidad de superficie específica, superando la actividad de MnOx, seguido de Cu1Mn1 y Cu2Mn1. 
Como se observa en la figura 11, las muestras mixtas de mayor actividad fueron Cu1Mn2 y $\mathrm{Cu} 1 \mathrm{Mn} 3$, aunque no superaron en ningún caso la actividad del óxido $\mathrm{MnOx}$. Con fines de comparación se presenta en la figura 12, las actividades de los catalizadores preparados por los dos métodos, como se aprecia la muestra simple $\mathrm{MnOx}$ y las mixtas Cu1Mn2-método EG y Cu1Mn3-método CIT fueron las más activas. En todos los casos, la introducción del Mn en la estructura inicial del $\mathrm{CuOx}$ permitió mejorar la actividad de manera significativa, tal como se observa en la tabla 4. Las menores temperaturas $\mathrm{T}_{95 \%}$ y máxima cantidad de COV convertido a $250^{\circ} \mathrm{C}$ corresponden a las muestras mixtas $\mathrm{Cu} 1 \mathrm{Mn} 2$-método EG y Cu1Mn3-método CIT.

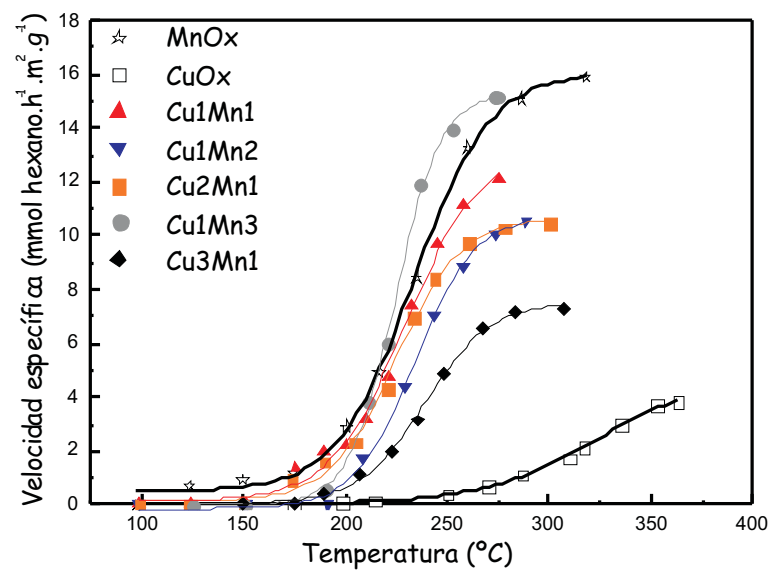

Figura 10. Curvas de velocidad específica de los catalizadores sintetizados por el método CIT.

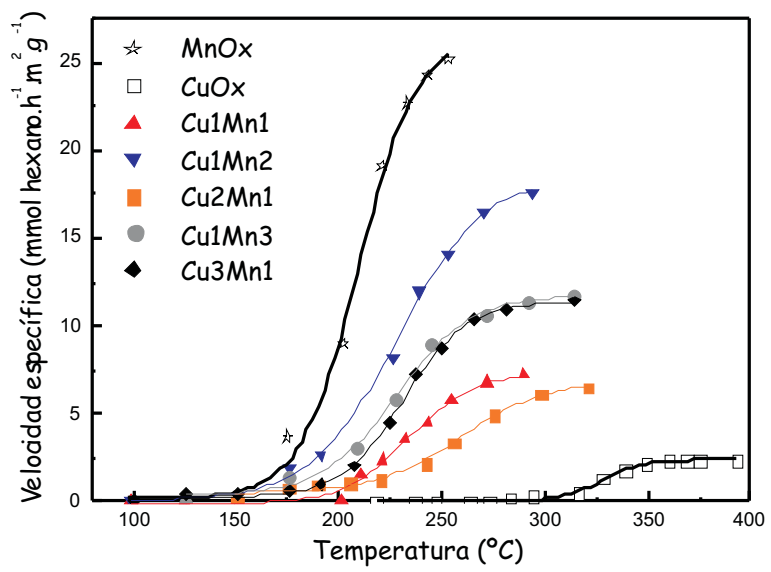

Figura 11. Curvas de velocidad específica de los catalizadores sintetizados por el método EG. 


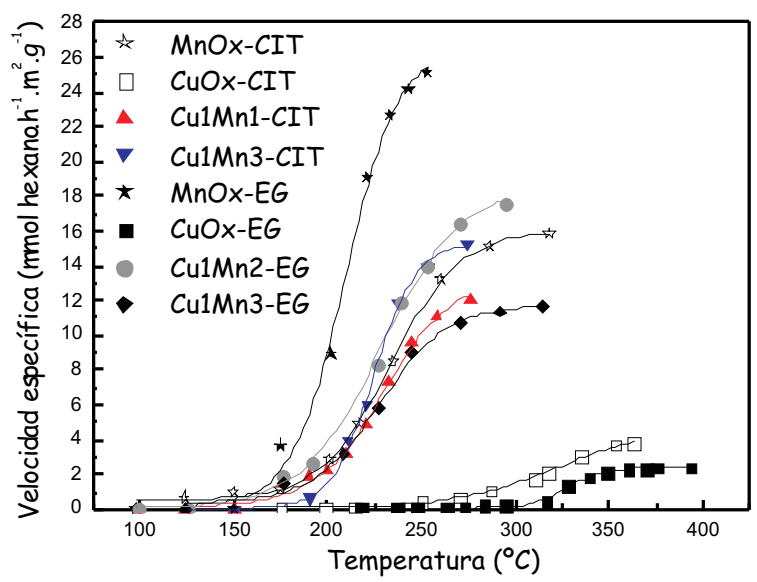

Figura 12. Comparación de la velocidad específica de los catalizadores sintetizados por el método CIT con los preparados por el método EG.

Tabla 4. Temperaturas $\mathrm{T}_{50 \%}$ y $\mathrm{T}_{95 \%} \mathrm{y}$ cantidad de milimoles de $\mathrm{n}$-hexano convertidos a $250^{\circ} \mathrm{C}$ de los catalizadores de $\mathrm{Cu}-\mathrm{Mn}$ en la combustión de n-hexano.

\begin{tabular}{|c|ccc|ccc|}
\hline Catalizador & \multicolumn{3}{|c|}{ Método CIT } & \multicolumn{3}{c|}{ Método EG } \\
& $\mathbf{T}_{\mathbf{5 0}}\left({ }^{\mathbf{0}} \mathbf{C}\right)$ & $\mathbf{T}_{\mathbf{9 5}}\left({ }^{\mathbf{0}} \mathbf{C}\right)$ & mmol-hex & $\mathbf{T}_{\mathbf{5 0}}\left({ }^{\mathbf{0}} \mathbf{C}\right)$ & $\mathbf{T}_{\mathbf{9 5}}\left({ }^{\mathbf{0}} \mathbf{C}\right)$ & m mol-hex \\
CuOx & 311,7 & 356,3 & 0,33 & 328,2 & 393,0 & 0 \\
$\mathrm{Cu} 3-\mathrm{Mn} 1$ & 238,3 & 279,2 & 5,0 & 227,1 & 287,1 & 8,7 \\
$\mathrm{Cu} 2-\mathrm{Mn} 1$ & 224,7 & 263,8 & 8,8 & 254,6 & 306,5 & 2,9 \\
$\mathrm{Cu} 1-\mathrm{Mn} 1$ & 227,5 & 270,0 & 10,0 & 233,9 & 278,9 & 5,4 \\
$\mathrm{Cu} 1-\mathrm{Mn} 2$ & 234,5 & 277,7 & 8,0 & 227,1 & $\mathbf{2 7 7 , 4}$ & $\mathbf{1 3 , 6}$ \\
$\mathrm{Cu} 1-\mathrm{Mn} 3$ & 224,7 & $\mathbf{2 6 1 , 7}$ & $\mathbf{1 3 , 8}$ & 230,9 & 278,9 & 9,0 \\
$\mathrm{MnOx}$ & 232,2 & 287,5 & 11,5 & 208,5 & 243,9 & 25,0 \\
\hline
\end{tabular}

Los resultados revelan que la superficie específica ha sido un factor importante pues las muestras mixtas con mayor superficie mostraron las actividades más altas como el caso de los catalizadores Cu1-Mn3-método CIT y Cu1-Mn2-método EG (tabla 4). La presencia simultánea del Mn en la estructura del CuOx permitió mejorar la actividad de las muestras, probablemente debido a la estructura hopcalita que permite el establecimiento del equilibrio: $\mathrm{Cu}(\mathrm{II})+\mathrm{Mn}(\mathrm{III})=\mathrm{Cu}(\mathrm{I})+\mathrm{Mn}(\mathrm{IV})$, que se sustenta en la presencia de $\mathrm{Mn}(\mathrm{III})$ y $\mathrm{Mn}(\mathrm{IV})^{23}$, confirmada por el análisis XRD.

\section{CONCLUSIONES}

- Se prepararon catalizadores en base a óxidos mixtos de $\mathrm{Cu}-\mathrm{Mn}$ variando la relación $\mathrm{Cu} / \mathrm{Mn}$ entre los metales de 0,33 a 3; con fines comparativos se prepararon además los óxidos simples de estos metales.

- Los resultados del área superficial de los catalizadores revelaron que el óxido simple de manganeso $(\mathrm{MnOx})$ fue el de mayor área. Por difracción de rayos X, se detectó que los 
catalizadores de óxidos mixtos presentaban los picos característicos correspondientes a los compuestos tipo espinela-hopcalita $\left(\mathrm{CuMn}_{2} \mathrm{O}_{4} \mathrm{y} \mathrm{Cu}_{1.5} \mathrm{Mn}_{1.5} \mathrm{O}_{4}\right)$.

- Todos los catalizadores ensayados permitieron la combustión total de n-hexano. La actividad medida como velocidad específica, por unidad de tiempo y por gramo de catalizador indicó que los catalizadores de $\mathrm{Cu}-\mathrm{Mn}$ independiente del método de preparación convierte totalmente el n-hexano a temperaturas menores de $300^{\circ} \mathrm{C}$. Los resultados mostraron que la superficie específica fue un factor importante, evidenciado por las superficies más altas obtenidas por los catalizadores Cu1Mn2 (método EG) y Cu1Mn3 (método CIT), además de una estructura tipo hopcalita.

\section{AGRADECIMIENTO}

Los autores agradecen al Instituto General de Investigación (IGI) de la Universidad Nacional de Ingeniería por el apoyo económico concedido para la realización de este trabajo durante el año 2014.

\section{REFERENCIAS}

1. Bastos S.S.T., Orfao J.J.M., Freitas M.M.A., Pereira M.F.R., Figuereido J.L., Manganese oxide catalysts synthesized by exotemplating for the total oxidation of etanol, Appl. Catal. B 2009; 93: 30-37.

2. Li W.B., Zhuang M., Wang J.X., Catalytic combustion of toluene on Cu-Mn/MCM-41 catalysts: Influence of calcination temperature and operating conditions on the catalytic Catal. Today 2008; 137: 340-344.

3. Kim S.C., Shim W.G., Catalytic combustion of VOCs over a series of manganese oxide catalysts, Appl. Catal. B 2010; 98: 180-185.

4. Perez H. Navarro P., Torres G., Sanza O., Montes M., Evaluation of manganese OMSlike cryptomelane supported on SBA-15 in the oxidation of ethyl acetate, Catal. Today 2013;212: 149- 156.

5. Perez H. Navarro P., Delgado J.J., Montes M., Mn-SBA15 catalysts prepared by impregnation: Influence of the manganese precursor, Appl. Catal. A 2011; 400: 238-248

6. Santos V.P., Pereira M.F.R., Órfão J.J.M., Figueiredo J.L., The role of lattice oxygen on the activity of manganese oxides towards the oxidation of volatile organic compounds, Appl. Catal. B 2010; 99: 353-363.

7. Barroso Quiroga M., Barbero B., Cadus L., Synthesis of a catalyst of Mn-Fe-O by mechano-chemical reaction, Appl. Catal. A 2014; 474: 26-33.

8. Azalim S., Brahmi R., Agunaou M., Beaurain A., Giraudon J.M., Lamonier J.F., Washcoating of cordierite honeycomb with $\mathrm{Ce}-\mathrm{Zr}-\mathrm{Mn}$ mixed oxides for VOC catalytic oxidation, Chem. Eng. J. 2013; 223: 536-546.

9. Azalim S., Franco M., Brahmi R., Giraudon J.M., Lamonier J.F., Removal of oxygenated volatile organic compounds by catalytic oxidation over $\mathrm{Zr}-\mathrm{Ce}-\mathrm{Mn}$ catalysts, J. Hazard. Mater. 2011; 188: 422-427.

10. Ludvíková J., Jirátová K,. Klempa J., Boehmová V., Obalová L, Titania supported Co-Mn-Al oxide catalysts in total oxidation of ethanol, Catal. Today 2012; 179: 164169.

11. Hosseini S.A., Niaei A., Salari D., Nabavi S.R., Nanocrystalline $\mathrm{AMn}_{2} \mathrm{O}_{4}(\mathrm{~A}=\mathrm{Co}, \mathrm{Ni}$, $\mathrm{Cu})$ spinels for remediation of volatile organic compounds - synthesis, characterization and catalytic performance, Ceram. Int. 2012; 38: 1655-1661. 
12. Buciuman F.C., Patcas F., Hahn T., A spillover approach to oxidation catalysis over copper and manganese mixed oxides, Chem. Eng. Process. 1999; 38: 563-569.

13. Tanaka Y., Takeguchi T., Kikuchi R., Eguchi K., Influence of preparation method and additive for $\mathrm{Cu}-\mathrm{Mn}$ spinel oxide catalyst on water gas shift reaction of reformed fuels, Appl. Catal. A 2005; 279: 59-66.

14. Picasso G., Sun Kou M.R., Salazar I., López A., Síntesis de catalizadores nanoestructurados basados en óxido de Mn para la eliminación de n-hexano, Revista de la Sociedad Química del Perú 2011; 77 (1): 11-26.

15. Picasso G., M. R. Sun Kou, G. Gómez, E. Hermoza, M. P. Pina, J. Herguido, Nanocatalizadores basados en óxidos de Fe para la combustión catalítica de n-hexano, Revista de la Sociedad Química del Perú 2009; 75 (2): 163-176.

16. Picasso G., Sun Kou M.R., Zavala C., Cruz R., López A., Preparación de catalizadores basados en óxidos mixtos de Ce/Mn por coprecipitación para la combustión de nhexano, Revista de la Sociedad Química del Perú 2013; 79(4):367-380.

17. Behar S., Gonzales P., Agulhon P., Quignard F., Swierczynski D., New synthesis of nanosized Cu-Mn spinels as efficient oxidation catalysts, Catal. Today 2012; 189: 3541.

18. Seyyed Ebrahimi S.A., Masoudpanah S.M., Effects of $\mathrm{pH}$ and citric acid content on the structure and magnetic properties of Mn $\mathrm{Zn}$ ferrite nanoparticles synthesized by a sol-gel autocombustion method, J. Magn. Magn. Mater. 2014; 357: 77-81.

19. Ahmad M., Ali I., Grössinger R., Kriegisch M., Kubel F., Rana M.U., Effects of divalent ions substitution on the microstructure, magnetic and electromagnetic parameters of $\mathrm{Co}_{2} \mathrm{~W}$ hexagonal ferrites synthesized by sol-gel method, J. Alloys Comp. 2013; 579: 57-64.

20. Hosseini S.A., Sadeghi M.T., Alemi A., Aligholi Niael A., Salarr D., Kafi-Ahmadi L., Synthesis, Characterization, and Performance of $\mathrm{LaZn}_{\mathrm{x}} \mathrm{Fe}_{1-\mathrm{x}} \mathrm{O}_{3}$ Perovskite Nanocatalysts for toluene combustion, Chin. J. Catal.2010;31 (7): 747-750.

21. Szczygieł I., Winiarska K., Bienko A., Suracka K., Gaworska-Koniarek D., The effect of the sol-gel autocombustion synthesis conditions on the $\mathrm{Mn}-\mathrm{Zn}$ ferrite magnetic properties, J. Alloys Comp. 2014; 604: 1-7.

22. Mangalaraja R.V., Ananthakmar S., Manohara P., Gnanama F.D., Awano M., Characterization of $\mathrm{Mn}_{0.8} \mathrm{Zn}_{0.2} \mathrm{Fe}_{2} \mathrm{O}_{4}$ synthesized by flash combustion technique, J. Mater. Sci. Eng. A 2004; 367: 301-305.

23. Hosseini, S.A., Niaei A., Salari D., Alvarez-Galvan M.C., Fierro J.L.G., Study of correlation between activity and structural properties of $\mathrm{Cu}-(\mathrm{Cr}, \mathrm{MnandCo})_{2}$, nano mixed oxides in VOC combustion, Ceramics International 2014; 40: 6157-6163. 